\title{
Nucleosynthesis of s-elements in zero-metal AGB stars
}

\author{
S. Goriely and L. Siess
}

Institut d'Astronomie et d'Astrophysique, Université Libre de Bruxelles, CP 226, 1050 Brussels, Belgium

Received 24 July 2001 / Accepted 6 September 2001

\begin{abstract}
Contrary to previous expectations, recent evolutionary models of zero-metallicity stars show that the development of mixing episodes at the beginning of the AGB phase allows low- and intermediate-mass stars to experience thermal pulses. If these stars, like their metal-rich counterparts, also experience partial mixing of protons from the H-rich envelope into the C-rich layers at the time of the third dredge-up, an extensive neutron capture nucleosynthesis leads to the production of s-process nuclei up to $\mathrm{Pb}$ and $\mathrm{Bi}$. Nucleosynthesis calculations based on stellar AGB models are performed assuming a parameterized $\mathrm{H}$-abundance profile below the convective envelope at the time of the third dredge-up. Despite the absence of Fe-group elements, the large neutron flux resulting from the ${ }^{13} \mathrm{C}(\alpha, \mathrm{n}){ }^{16} \mathrm{O}$ reaction leads to an efficient production of s-process elements starting from the neutron captures on the C-Ne isotopes. Provided partial mixing of protons takes place, it is shown that population III AGB stars should be enriched in s-process elements and overall in $\mathrm{Pb}$ and $\mathrm{Bi}$.
\end{abstract}

Key words. nucleosynthesis - stars: AGB - abundances

\section{Introduction}

The determination of chemical abundances at the surface of evolved stars provides important clues on their structural evolution and traces the hidden processes taking place in their nuclearly active interior. Asymptotic giant branch (AGB) stars, and in particular zero-metallicity stars, represent an ideal laboratory for such a study. They correspond to the late phase of the evolution of stars with masses between about 1 and $8 M_{\odot}$, which includes more than $80 \%$ of all stars; they exhibit peculiar surface abundances as compared to other red giant stars; and many of them are characterized by a strong mass loss (up to $10^{-4} M_{\odot} \mathrm{yr}^{-1}$ ) which eject the surface material into the interstellar medium, contributing thereby to the galactic chemical evolution. Among those, the population III stars, i.e. the first to form with the chemical composition inherited from the Big Bang nucleosynthesis, are of particular interest to understand the early enrichment of the universe in elements heavier than hydrogen and helium. Little is known observationally on population III stars, since so far no star of population III initial composition has yet been found. If s-process enriched AGB stars are commonly observed nowadays, they all correspond to metallicities $[\mathrm{Fe} / \mathrm{H}] \gtrsim-3$. However, the detection of carbon and nitrogen enrichments in the intergalactic medium at high redshifts and in a considerable number of extremely metal-poor stars of our Galaxy suggests the existence of primordial AGB stars (see e.g Abia et al. 2001).

The abundance peculiarities observed at the surface of AGB stars are understood as resulting from the mixing

Send offprint requests to: S. Goriely,

e-mail: sgoriely@astro.ulb.ac.be of material synthesized in the interior with the surface layers as the convective envelope deepens during the socalled third dredge-up (denoted hereafter 3DUP). But the nucleosynthesis of s-process elements requires neutron-rich environments that cannot be accounted for by the sole action of the dredge-up. One way to solve the problem is to assume the partial mixing $(\mathrm{PM})$ of protons from the envelope into the ${ }^{12} \mathrm{C}$-rich layers during a 3DUP event (Iben \& Renzini 1982; Busso et al. 1999). The incomplete operation of the CN cycle forms a " $13 \mathrm{C}$ pocket" which burns radiatively and releases a large number of neutrons. However, AGB models are still subject to large uncertainties concerning the consistent prediction of both the 3DUP and PM processes (e.g. Mowlavi 1999). In particular, the 3DUP and PM properties are sensitive to the stellar characteristics (such as the mass, metallicity or mass loss rate) and to the numerical scheme used to describe the convective boundaries (e.g. Frost \& Lattanzio 1996). In the past years, the mixing scenario has been revived by the incorporation of new physical ingredients to model overshooting (Herwig et al. 1999) and rotation (Langer et al. 1999). Models including these new treatments predict the formation of a ${ }^{13} \mathrm{C}$ pocket in a zone comprising some $10^{-5} M_{\odot}$. However, the total amount of ${ }^{13} \mathrm{C}$ produced in this way depends on the PM parameters (extent and efficiency of the overshoot, amplitude of rotation) which cannot be quantified ab initio at the present time. In this context, the resulting uncertainties on the subsequent nucleosynthesis can only be handled at the moment in a parametric way.

Recently several works have been devoted to the study of the structure and evolution of primordial stars (Siess et al. 2001; Chieffi et al. 2001; Marigo et al. 2001). However, from a nucleosynthesis point of view, 
the production of elements heavier than $\mathrm{Mg}$ and ultimately of iron in low- and intermediate-mass population III stars remains largely a mystery. Assuming that primordial AGB stars suffer PM of protons as their higher metallicity counterparts do, we analyze its effect on the resulting nucleosynthesis applying the PM model developed by Goriely \& Mowlavi (2000) to the realistic 1-D AGB models computed by Siess et al. (2001). The corresponding modeling is described in Sect. 2. In Sect. 3, we study in detail the nucleosynthesis operating in the PM zone and its consequences on the surface abundance predictions of zero-metallicity AGB stars. In particular, it is shown that the initial absence of Fe does not prevent the formation of the highest-mass elements and leads to the overproduction of $\mathrm{Pb}$ and $\mathrm{Bi}$.

\section{Astrophysical model and input physics}

The stellar models used in this study are part of a recent grid computed by Siess et al. (2001) and we refer the reader to this publication for ampler details. Among the available mass tracks, we selected the $3 M_{\odot}$ model for two main reasons: it is our most advanced model which accounts for 22 thermal pulses and $3 M_{\odot}$ is a somewhat "representative" mass for an AGB star, between the low$\left(1 M_{\odot}\right)$ and massive $\left(8 M_{\odot}\right)$ ones. Briefly, the evolution of this model (which lasts for about $250 \mathrm{Myr}$ ) proceeds as follows: during the main sequence, the star cannot activate the CNO cycle because of the absence of ${ }^{12} \mathrm{C}$. Consequently, it contracts and develops higher central temperatures to sustain its energy budget by the poorly efficient p-p chains. When the central temperature reaches $10^{8} \mathrm{~K}$, the $3 \alpha$ reactions start and carbon is produced in the core. The CNO cycle finally activates when the ${ }^{12} \mathrm{C}$ mass fraction exceeds $\sim 10^{-11}$. At the exhaustion of hydrogen in the center, the $3 \alpha$ reactions are still effective and the star enters the core He burning phase without experiencing the first dredge-up. The beginning of the AGB phase of zero-metallicity stars is characterized by the occurrence of mixing episodes which enables the carbon produced in the He burning shell to mix with the envelope. This carbon pollution of the structure activates the CNO cycle in the $\mathrm{H}$ burning shell and allows primordial stars to resume a "standard" AGB evolution comparable to that of more metal rich stars. Note that without this mixing episode, the $3 M_{\odot}$ models would probably not suffer thermal pulses (and 3DUP). Primordial AGB stars are characterized by massive $\mathrm{CO}$ cores as a result of initially expanding their nuclear core to compensate for the non-activation of the CNO cycle. They also rapidly undergo third dredge-up events (after 9 pulses in this case) and exhibit higher temperatures in the pulse compared to more metal-rich stars. Of nucleosynthetic relevance is the activation of very efficient hot bottom burning (HBB) after the 20th pulse, where the temperature at the base of the convective envelope reaches $\sim 8 \times 10^{7} \mathrm{~K}$. The impact of $\mathrm{HBB}$ on the surface abundances of CNO elements is discussed in details in Siess et al. (2001). Finally, because of the low metal content in the envelope, mass loss is rather inefficient and by the end of the computations only $10^{-3} M_{\odot}$ is lost. Nevertheless, the successive dredge up episodes will increase little by little the opacity in the envelope and consequently the mass loss efficiency.

The temperature and density profiles given by the stellar evolution code are used as input data to compute the detailed nucleosynthesis resulting from the PM of protons. Protons from the envelope are ingested artificially from the bottom of the convective envelope into the underlying C-rich region at the time of the 3DUP events. We adopt two different $\mathrm{H}$-abundance profiles. The first one decreases exponentially with depth and ranges from $X_{\mathrm{p}}^{\text {mix }}=0.7$ at the bottom of the convective envelope to $10^{-6}$ at the bottom of the PM zone. The second $\mathrm{H}$ abundance profile decreases slower with depth than the previous case. It corresponds to the "Slow" curve in Fig. 10 of Goriely \& Mowlavi (2000). However, since the nucleosynthesis calculations are found to be almost identical in both cases, we present here only results using the second $\mathrm{H}$ abundance profile which can be considered as more characteristic of diffusion processes (Herwig et al. 1999). We consider a PM zone of extension $\lambda_{\mathrm{pm}}=0.05$ corresponding to $5 \%$ in mass of the pulse-driven convection zone. This corresponds to a zone comprising some $10^{-5} M_{\odot}$ subject to the s-process neutron irradiation.

The nucleosynthesis resulting from the ingestion of the protons into the C-rich layers at the time of each 3DUP is followed during the first 22 interpulse and pulse phases of the $3 M_{\odot}$ stellar model. In contrast to Goriely \& Mowlavi (2000), the ashes of the interpulse and pulse nucleosynthesis are reingested in the next interpulse/pulse sequence, so that multiple irradiations on former nucleosynthetic products are consistently taken into account. The nuclear reaction network includes 817 nuclei up to Po, and all relevant nuclear, weak and electromagnetic interactions. Note that the network needs to be extended to the neutronrich region because of the very large neutron densities generated in the late He-flashes. The latest experimental and theoretical reaction rates are taken from the Nuclear Astrophysics Library of the Brussels University (available at http://www-astro.ulb.ac.be). More details can also be found in Goriely \& Mowlavi (2000).

\section{The neutron capture nucleosynthesis}

To analyse the nucleosynthetic impact of the PM of protons into the $\mathrm{C}$-rich region, we first consider one interpulse/pulse/3DUP phase. The richest nucleosynthesis occurs during the interpulse phase. More specifically, for low proton-to-carbon ratios $\left[Y_{\mathrm{p}} / Y\left({ }^{12} \mathrm{C}\right) \lesssim 1\right.$, where $Y(A)=$ $X(A) / A$ is the abundance of $A$ by number], neutrons are efficiently produced after ${ }^{13} \mathrm{C}$ has been synthesized, and subsequently destroyed by ${ }^{13} \mathrm{C}(\alpha, \mathrm{n}){ }^{16} \mathrm{O}$ (Fig. 1$)$. In the absence of Fe-group nuclei, neutrons are captured by all the abundant $\mathrm{C}, \mathrm{N}, \mathrm{O}, \mathrm{F}$ and $\mathrm{Ne}$ isotopes and enable the production of high mass elements. The fast $(\mathrm{n}, \alpha)$ reaction on ${ }^{33} \mathrm{~S}$ forms a major bottle-neck braking down 
the s-process flow partially. As soon as the bottle-neck is passed, radiative neutron captures followed by $\beta$-decays on unstable nuclei lead to the synthesis of all medium and heavy elements up to $\mathrm{Pb}$ and $\mathrm{Bi}$. The ${ }^{33} \mathrm{~S}(\mathrm{n}, \alpha){ }^{30} \mathrm{Si}$ recycling reduces the total amount of s-elements produced by a factor of about 5 . The evolution of the mass fraction of some representative nuclei (with a magic number of neutrons $N=28,50,82$ and 126) is displayed in Fig. 1. In the series of neutron captures and $\beta$-decays characteristic of the s-process, iron group nuclei do not play any particular role, in contrast to the classical picture of the s-process where the neutron captures take place on the abundant ${ }^{56}$ Fe mainly.

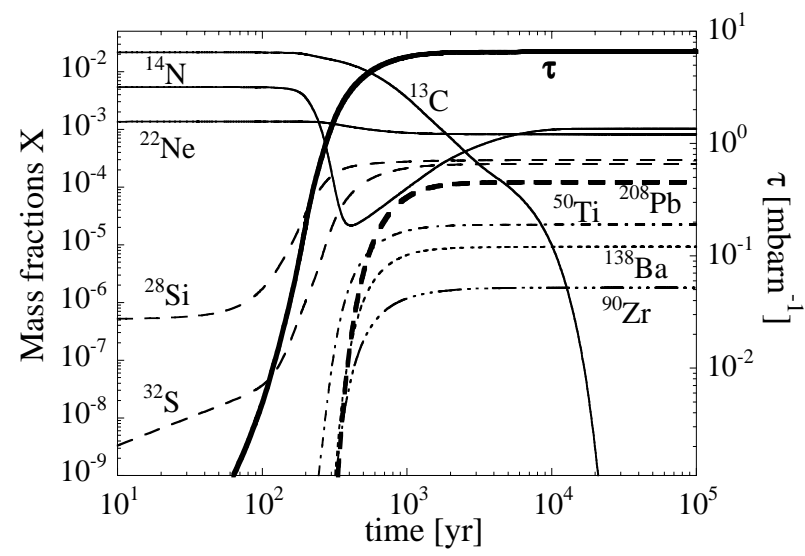

Fig. 1. Mass fraction of some elements representative of the neutron capture nucleosynthesis taking place in the PM layer characterized by an initial proton-to- ${ }^{12} \mathrm{C}$ ratio of $10^{-2}$ during the 21st interpulse phase of the $M=3 M_{\odot}, Z=0$ AGB star. Also given (bold solid line) is the time dependence of the neutron exposure $\tau$.

For $10^{-2} \lesssim Y_{\mathrm{p}} / Y\left({ }^{12} \mathrm{C}\right) \lesssim 1$, the neutron exposure $\tau=\int_{0}^{t} N_{\mathrm{n}} v_{T} \mathrm{~d} t$ reaches $10 \mathrm{mbarn}^{-1}\left(v_{T}\right.$ is the most probable relative neutron-nucleus velocity at temperature $T$ ) and the neutron density $N_{\mathrm{n}}$ a few $10^{9} \mathrm{~cm}^{-3}$. These high values explain the large production of metals up to $\mathrm{Pb}$ and $\mathrm{Bi}$ despite the absence of initial iron (Fig. 2a). For relatively high proton-to- ${ }^{12} \mathrm{C}$ abundance ratios $\left[Y_{\mathrm{p}} / Y\left({ }^{12} \mathrm{C}\right) \gtrsim 1\right],{ }^{14} \mathrm{~N}$ is more abundant than ${ }^{13} \mathrm{C}$ and prevents any significant neutron production. In this case, the PM of protons only affects light species. The fast ${ }^{14} \mathrm{~N}(\mathrm{n}, \mathrm{p}){ }^{14} \mathrm{C}$ reaction is responsible for the production of ${ }^{14} \mathrm{C}$ and later of ${ }^{18} \mathrm{O}$ by ${ }^{14} \mathrm{C}(\alpha, \gamma){ }^{18} \mathrm{O}$. This reaction also releases a large amount of protons which are captured by ${ }^{18} \mathrm{O}$ to form ${ }^{15} \mathrm{~N}$. Subsequently, in the pulse-driven convection zone, ${ }^{19} \mathrm{~F}$ is largely produced by ${ }^{15} \mathrm{~N}(\alpha, \gamma){ }^{19} \mathrm{~F}$. However, as soon as envelope HBB is activated, the dredged-up ${ }^{19} \mathrm{~F}$ is destroyed. $\mathrm{HBB}$ also increases the surface abundance of ${ }^{14} \mathrm{~N}$ and ${ }^{23} \mathrm{Na}$ significantly at the expense of CNO isotopes and ${ }^{22} \mathrm{Ne}$, respectively.

As the core mass grows, the temperature in the thermal pulse increases and by the 20th pulse, it exceeds $4 \times 10^{8} \mathrm{~K}$. In these conditions, ${ }^{22} \mathrm{Ne}$ is rapidly burnt by ${ }^{22} \mathrm{Ne}(\alpha, \mathrm{n}){ }^{25} \mathrm{Mg}$ and additional neutrons are released.

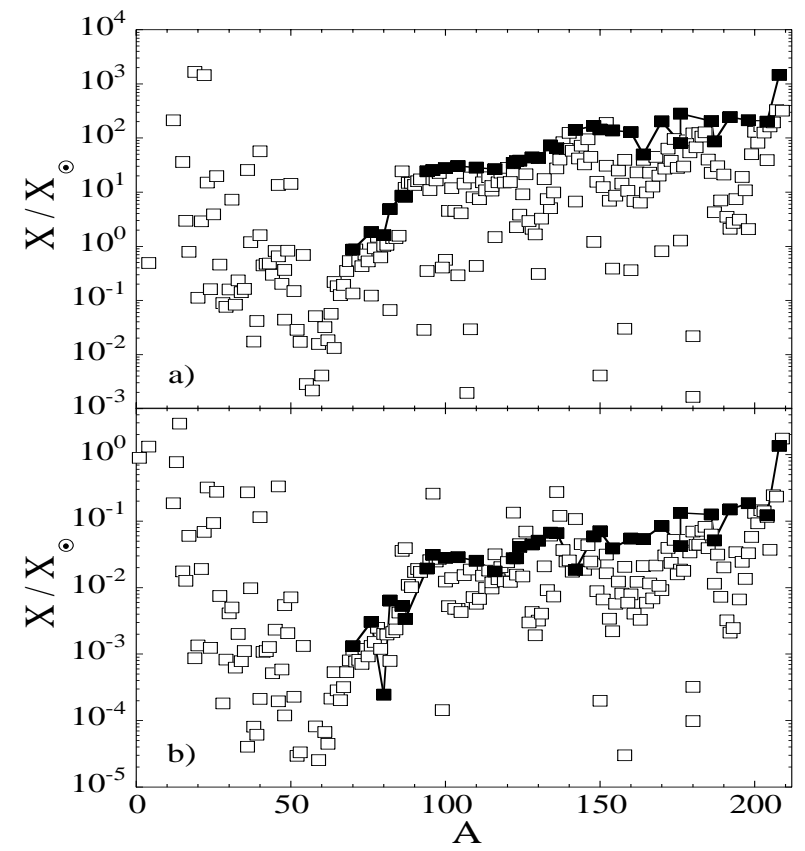

Fig. 2. Abundance distribution (relative to solar) at the end of the 21st interpulse phase a) and following the next pulse b) for the $M=3 M_{\odot}, Z=0$ AGB star. In panel a), the distribution is averaged over the mass range covered by the PM region (in this case $M_{\mathrm{pm}}=4 \times 10^{-4} M_{\odot}$ ) while case $\mathbf{b}$ ) gives the surface abundance after the 3DUP. Full squares correspond to s-only isotopes. Note the nuclear rearrangement of some s-only elements during the pulse.

In the 22nd pulse, the neutron density reaches $N_{\mathrm{n}}=$ $10^{15} \mathrm{~cm}^{-3}$, but for a short period of time and mostly near the bottom of the He-flash zone. Consequently, the pulse-averaged resulting neutron exposure is small. Nevertheless, the neutron burst allows for some rearrangements of the abundance distribution of peculiar s-only isotopes (e.g. ${ }^{80} \mathrm{Kr}$ or ${ }^{142} \mathrm{Nd}$, see Fig. 2b) and of some sr-nuclei by short-cutting the s-process flow. In particular, ${ }^{122} \mathrm{Sn},{ }^{136} \mathrm{Xe},{ }^{137} \mathrm{Ba}$ and ${ }^{142} \mathrm{Ce}$ are overproduced compared with neighboring isotopes. Abundances of the so-called $T$-dependent s-branching nuclei, e.g. ${ }^{164} \mathrm{Er}$, are also modified because of the high temperatures prevailing in the pulse. Among the medium-mass nuclei, ${ }^{36} \mathrm{~S},{ }^{40} \mathrm{Ar}$ and ${ }^{46} \mathrm{Ca}$ are largely produced. Note that the large dip in the $X / X_{\odot}$ scale observed in Figs. 2 and 3 for the Fe-group elements is due to their high solar values, and consequently small abundances relative to solar.

Except for the first 6 interpulse/pulse sequences where the $\mathrm{C}$ abundance left over by the preceding pulse-driven convective zone is small, the nucleosynthesis resulting from the PM of protons is found to be qualitatively identical from one interpulse/pulse phase to the other (the only significant difference originates from the temperatures reached at the bottom of the He-flash zone). The relatively small extent of the PM zone $\left(\lambda_{\mathrm{pm}}=0.05\right)$ does not allow a significant cumulative neutron irradiation on the ashes of the previously synthesized material. As a consequence, the most abundant material, namely the 
elements comprised between $\mathrm{C}$ and $\mathrm{Ne}$, always remain the seed nuclei for the neutron captures in the PM zone.

The above-described nucleosynthesis is in many respects similar to the one found in the low-metallicity $(Z=0.001)$ star subject to the same mixing of protons (Goriely \& Mowlavi 2000). In the radiative C-rich layers during the interpulse phase, the overall abundance distributions are much the same even if the seed nuclei at the origin of the heavy elements are in both cases quite different. For stars with metallicities $Z \lesssim 0.001$, the number of neutrons available per seed nuclei exceeds the number needed to produce the heaviest s-nuclei, i.e. $\mathrm{Pb}$ and $\mathrm{Bi}$, so that the type of seeds as well as their initial abundances only affect the absolute abundance of heavy elements synthesized by the s-process, but not their relative distribution. However, for extremely low metallicity stars, the higher temperatures reached at the bottom of the pulsedriven convective zones can reshape the s-abundance distribution differently.

Provided partial mixing of protons occurs in population III stars in a similar way as in more metal-rich stars, the primordial stars should exhibit a strong overabundance of $\mathrm{Pb}$ and $\mathrm{Bi}$ compared with other s-process elements $([\mathrm{Pb} / \mathrm{s}] \gtrsim 1)$. Therefore, such stars could be dubbed population III Pb-stars (Goriely \& Mowlavi 2000; Van Eck et al. 2001). In addition to their large overabundance in ${ }^{14} \mathrm{~N}$ (mainly due to the HBB transmuting all the CNO envelope isotopes into ${ }^{14} \mathrm{~N}$ ), these $\mathrm{Pb}$-stars are also predicted to have a large ${ }^{23} \mathrm{Na}$ content, as shown in Fig. 3. Van Eck et al. (2001) observed low-metallicity stars (down to $[\mathrm{Fe} / \mathrm{H}]=-2.45)$ enriched in s-process elements and characterized by large $\mathrm{Pb}$ overabundances $([\mathrm{Pb} / \mathrm{hs}] \gtrsim 1$, where hs denotes heavy s-elements such as Ba, La or Ce). The discovery of these $\mathrm{Pb}$-stars confirms the s-process efficiency in low-metallicity stars. However, this conclusion is not reached by Aoki et al. (2000) who found in a metalpoor s-process enriched stars $([\mathrm{Fe} / \mathrm{H}]=-2.7)$, a $\mathrm{Pb}$ overabundance $([\mathrm{Pb} / \mathrm{Fe}]=+2.6)$ but no $\mathrm{Pb}$-to-s overabundance $([\mathrm{Pb} / \mathrm{hs}] \simeq 0)$. This latter observation remains to be explained.

While the discovery of Pb-stars (Van Eck et al. 2001) strongly confirms the "proton-mixing" scenario for the detailed operation of the s-process in AGB stars, their is still no evidence neither on the existence of population III stars nor on the possible mixing of protons in such stars. For this reason, it would be premature at this stage to estimate the possible contribution of population III stars to the galactic enrichment in s-process elements, and more particularly in $\mathrm{Pb}$ (e.g. Travaglio et al. 1999).

\section{Conclusion}

Assuming that population III stars experience, just like more metal-rich stars, a partial mixing of protons from the H-rich envelope into the C-rich layers at the time of the third dredge-up, an extensive neutron capture nucleosynthesis is found to take place leading to the efficient production of s-process nuclei. Despite the absence

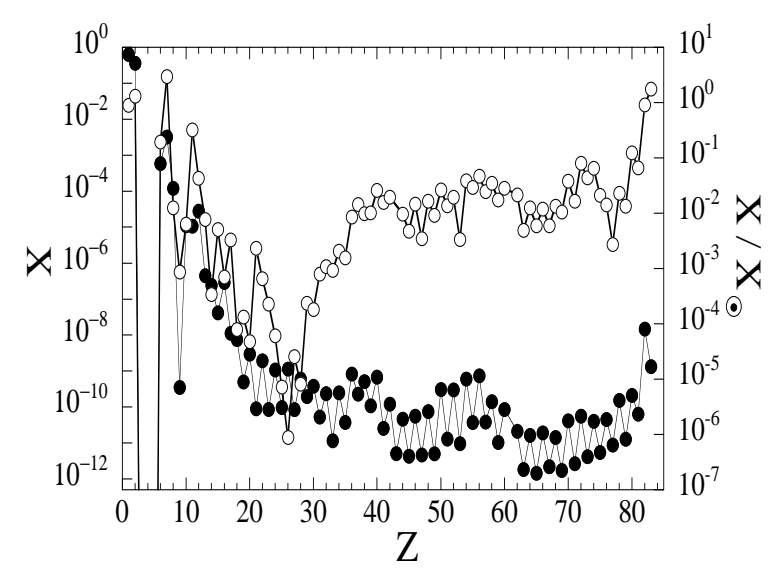

Fig. 3. Elemental abundances (full circles) and overabundances relative to solar (open circles) at the surface of the $M=3 M_{\odot}$ zero-metallicity AGB star after the 22nd convective pulse phase.

of Fe-group elements, the large neutron flux resulting from the ${ }^{13} \mathrm{C}(\alpha, \mathrm{n}){ }^{16} \mathrm{O}$ reaction transmutes part of the $\mathrm{C}-\mathrm{Ne}$ isotopes into s-nuclei with a large overabundance of $\mathrm{Pb}$ and Bi. Provided primordial stars are observable today, if they exhibit a surface enrichment in s-process elements, a strong overabundance of $\mathrm{Pb}$ and $\mathrm{Bi}$ compared with other s-process elements $([\mathrm{Pb} / \mathrm{s}] \gtrsim 1)$ is predicted. These population III Pb-stars remain to be observed. Their discovery or the indirect identification of their pollution in later stellar generations would prove that the galactic enrichment in s-process elements could have started already a few hundred million years after the Big Bang.

Acknowledgements. S. G. is FNRS Research Associate. L. S. benefits of a European TMR "Marie Curie" fellowship at ULB.

\section{References}

Abia, C., Dominguez, I., Straniero, O., et al. 2001, ApJ, 557, 126

Aoki, W., Norris, J. E., Ryan, S. G., et al. 2000, ApJ, 536, L97

Busso, M., Gallino, R., \& Wasserburg, G. J. 1999, ARA\&A, 37,239

Chieffi, A., Dominguez, I., Limongi, M., \& Straniero, O. 2001, ApJ, 554, 1159

Frost, C. A., \& Lattanzio, J. C. 1996, ApJ, 473, 383

Goriely, S., \& Mowlavi, N. 2000, A\&A, 362, 599

Herwig, F., Blöcker, T., \& Schönberner, D. 1999, in 191st IAU Symp., PASP, ed. T. Le Bertre et al., 41

Iben, I. Jr., \& Renzini, A. 1982, A\&A, 263, L23

Langer, N., Heger, A., Wellstein, S., \& Herwig, F. 1999, A\&A, 346, L37

Marigo, P., Girardi, L., Chiosi, C., \& Wood, P. R. 2001, A\&A, 371,152

Mowlavi, N. 1999, A\&A, 344, 617

Siess, L., Livio, M., \& Lattanzio, J. 2001, ApJ, submitted

Travaglio, C., Galli, D., Gallino, R., et al. 1999, ApJ, 521, 691

Van Eck, S., Goriely, S., Jorissen, A., \& Plez, B. 2001, Nature, 412,793 